\title{
Binarization Techniques used for Grey Scale Images
}

\author{
Puneet \\ GRDIET,BATHINDA \\ Department of Computer Science \& Engineering
}

\author{
Naresh Kumar Garg \\ GZSPTU Campus,BATHINDA \\ Department of Computer Science \& Engineering
}

\begin{abstract}
ABSTARCT
Image binarization is important step in the OCR (Optical Character Recognition). There are several methods used for image binarization recently, but there is no way to select single or best method which is used for all images. The main objective of this paper is to present the study on various existing binarization algorithms and compared their measurements.

This paper will act as guide for fresher's to start their work on binarization.
\end{abstract}

\section{Keywords:}

Binarization, Threshold value, SNR, OCR

\section{INTRODUCTION}

The Binarization Method converts the grey scale image ( 0 up to 256 gray levels) in to black and white image ( 0 or 1$)$. The result of OCR highly depends upon the binarization. The high quality binarized image can give more accuracy in character recognition as compared original image because noise is present in the original image [1]. In fact problem is that which binarization algorithm is appropriate for all images. The selection of most optimal binarization algorithm is difficult, because different binarization algorithm gives different performance on different data sets. This is especially true in the case of historical documents images with variation in contrast and illumination. The algorithms divide into two categories a) Global Binarization b) Local Binarization. The global binarization methods used single threshold value for whole image and the local binarization method where the threshold value calculated locally pixel by pixel or region by region. The figure (a) show the basic block diagram of binarization

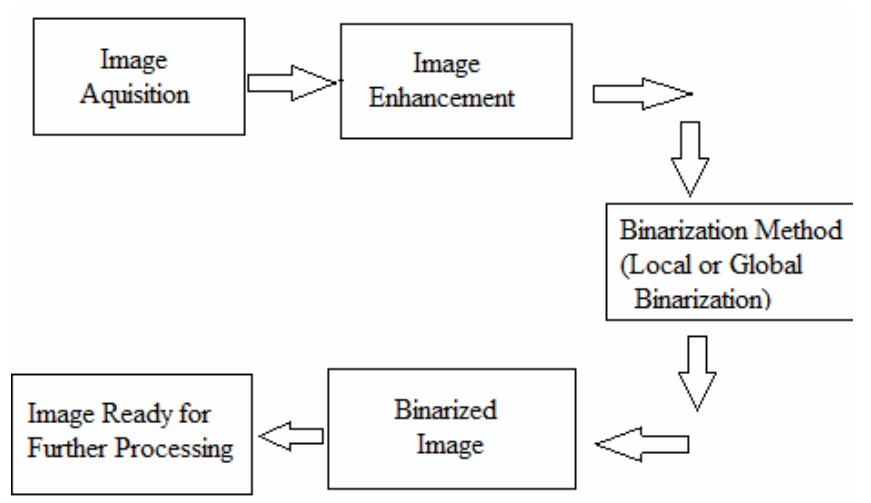

Fig.(a) Block Diagram of Binarization

\section{Binarization Methods}

This section classifies the some important local and global binarization methods that are currently used for binarization. For global binarization we choose:

1) Fixed Thresholding Method

2) Otsu Method

3) Kittler Method

For local binarization we choose:
1) Niblack Method
2) Adaptive Method
3) Sauvola Method
4) Bernsen Method

\subsection{Global Methods}

\subsubsection{Fixed Thresholding Method}

In Fixed Thresholding binarization method [2][3][4] fixed threshold value is used to assign 0's and 1's for all pixel positions in a given image. The basic idea for fixed binarization method is described as under.

$$
g(x, y)=\left\{\begin{array}{l}
1 \text { if } f(x, y)>=T \\
0 \text { otherwise }
\end{array}\right.
$$

T shows global threshold value. For various threshold values in fixed binarization methods the results are illustrated below.

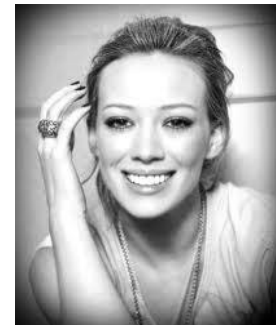

Original image

This paper is organized in following sections: Section 2 describes the different binarization methods. Section 3 describes the work done by different researcher. Section 4 presents the conclusion. 


\begin{tabular}{|c|c|}
\hline $\begin{array}{l}\text { THRESHOLD } \\
\text { VALUE }\end{array}$ & OUTPUT \\
\hline $\mathrm{T}=108$ & \\
\hline $\mathrm{T}=128$ & \\
\hline $\mathrm{T}=148$ & \\
\hline $\mathrm{T}=160$ & \\
\hline
\end{tabular}

TABLE-2.1

As shown in table 2.1 , outputs vary as given an input threshold value. From the above table it can be noticed that output at threshold $\mathrm{T}=160$ is better for this image. But $\mathrm{T}=160$ is not optimal threshold value for all the images. So it is very difficult to decide an optimal threshold value for a current input image. To overcome this difficulty we will discuss further more binarization techniques in which optimal threshold value is computed according to input image.

\subsubsection{Otsu Binarization Method}

In image processing, Otsu's thresholding method is used for automatic binarization level decision, based on the shape of the histogram [5]. Otsu's thresholding method involves iterating through all the possible threshold values and calculating a measure of spread for the pixel levels each side of the threshold, i.e. the pixels that either falls in foreground or background. The aim is to find the threshold value where the sum of foreground and background spreads is at its minimum.

\section{STEPS for Otsu Method}

1. Separate the pixels into two clusters according to the threshold.

$$
\begin{aligned}
& q 1(t)=\sum_{i=1}^{t} p(i) \quad \text { and } \quad q 2(t)= \\
& \sum_{i=t+1}^{I} p(i)
\end{aligned}
$$

$\mathrm{P}$ represents the image histogram.

2. Find the mean of each cluster.

$$
\begin{aligned}
& \text { 国 }(t)=\sum_{j=1}^{t} \frac{i p(i)}{q 1(t)} \text { and } \\
& \text { ?2(t) }=\sum_{j=t+1}^{I} \frac{i p(i)}{q 2(t)}
\end{aligned}
$$

3. Calculate the individual class variance.

$$
\sigma_{1}^{2}(t)=\sum_{i=1}^{t}[i-? 1(t)]^{2} \frac{p(i)}{q 1(t)}
$$

and

$$
\sigma_{2}^{2}(t)=\sum_{i=t+1}^{I}[i-\text { ?2 } 2(t)]^{2} \frac{p(i)}{q 2(t)}
$$

4. Square the difference between the means.

$$
\begin{aligned}
& \sigma_{b}^{2}(t)=\sigma^{2}-\sigma_{w}^{2}(t) \\
& =q 1(t)[1-q 1(t)][? 1(t)-? 2(t)]^{2}
\end{aligned}
$$

5. Finally, this expression can safely be maximized and the solution is $\mathbf{t}$ that is maximizing $\sigma_{b}^{2}(t)$

\subsubsection{Kilter and Illingworth Method}

The kilter method [6] is used mixture of Gaussian distribution to find threshold value. In kilter Method the $t$ is threshold that is used to segment the image into two parts background and foreground, both of the parts modelled by Gaussian distribution, $p_{B}(t)$ and $p_{F}(t)$, the $p_{\text {mix }}(t)$ mixture of these two Gaussian distribution.

$$
p_{\text {mix }}(t)=\alpha p_{B}(t)+(1-\alpha) p_{F}(t)
$$

Where $\alpha$ is determined by the portions of background and foreground in the image.

\subsection{Local Methods}

\subsubsection{Adaptive Binarization Method}

Adaptive binarization method [7] is used for local binarization. In this a window of $\mathrm{NxN}$ blocks slide over the entire image and threshold value is computed for each local area under the window for binarization. The adaptive method give more accurate result as compared to global binarization in such conditions where the 
image effected from bad shading, blurring, low resolution and non uniform illumination. In adaptive binarization any one of the technique like Niblack, Sauvola etc used to compute the local area threshold value.

\subsubsection{Niblack Method}

In Niblack method [8] the threshold value for the local area under the window is calculated pixel wise. The calculation of the threshold value is depending upon the local mean and standard deviation of window area. The threshold value is finding using following equation.

$$
\begin{gathered}
T_{\text {Niblack }}=m+k * s \\
T_{\text {Niblack }}=m+k \sqrt{\frac{1}{N P} \sum\left(p_{i}-m\right)^{2}}
\end{gathered}
$$

Where $\mathrm{m}$ is the mean of local area pixels of an image and $\mathrm{s}$ is the standard deviation of local pixel area. The value of $\mathrm{k}$ is fixed to 0.2 by the author.

\subsubsection{Sauvola Method}

The Sauvola algorithm [9] is a modified form of Niblack algorithm. It gives more performance than Niblack under such conditions as light variation on document image, light texture etc. In the Sauvola modification, the binarization is given by:

$$
T_{\text {sauvola }}=m *\left(1-k *\left(1-\frac{S}{R}\right)\right)
$$

Where $\mathrm{m}$ is the mean of pixels under window area, $\mathrm{S}$ is the dynamic range of variance and the value of $\mathrm{k}$ parameter may be in the range of $0-1$.

According to [10], the Sauvola method is better than Niblack method when the gray-level images convert to the black $\mathrm{n}$ white images. The author fix the $\mathrm{k}=0.5$ and $\mathrm{R}=128$.

\subsubsection{Bernsen method}

Bernsen is local binarization method which computes the threshold value from the pixel of image [11]. The equation used for calculation of threshold value is given below:

$$
T_{\text {Bernsen }}=\left(N_{\text {low }}+N_{\text {high }}\right) / 2
$$

Where $N_{\text {low }}$ and $N_{\text {high }}$ are the grey level values of the window.

\section{WORK DONE BY DIFFERENT RESEARCHERS}

The lot of work has been done in past years. The Aroop Mukherjee and Soumen Kanrar [12] have done the comparison study between the various algorithms based on Binarization algorithms and propose a methodologies for the validation of Binarization algorithms. In this work they have developed two novel algorithms to determine threshold values for the pixels value of the gray scale image. Chien-Hsing Chou, Wen-Hsiung Lin, Fu Chang [13] has proposed a novel binarization method for document images produced by cameras. The proposed method divides an image into several regions and decides to binaries each region by Otsu method. The decision rules are derived from a learning process that takes training images as input. Tests on images produced under normal and inadequate illumination conditions. Madhuri Latha.G, Chakravarthy.G [14] used Improved Bernsen Algorithm approaches for License Plate Recognition (LPR) applied to the intelligent transportation system. The proposed LPR algorithm consists of three modules: 1) Locating the license plates; 2) Segmenting the characters; and 3) Identifying the license characters. Zhang zhi-yong, song yang [15] has done the binarization of licence plate using Otsu in mat lab. Konstantin's Ntirogiannis, Basils Gatos and and Ioannis Pratikakis [16] addresses a pixel-based binarization evaluation methodology for historical and written/machine-printed document images. In the proposed evaluation scheme, they modified recall and precision evaluation measures using a weighting scheme that diminishes any potential evaluation bias. Yi Wang, Bin Fang and Li-Jun Lan [17] proposed mathematic morphology filter will be used as a low-pass filter to improve the illumination of license plate. To eliminate the impact of illumination, average filter, Gaussian filter and median filter are chosen to filter the plate image respectively. They convert the gray-scale image to binary image by the local threshold obtained from the convolved image with illumination compensation coefficient.

\section{CONCLUSION}

Global thresholding method is better approach for calculate the threshold values of a grey scale images. But it doesn't give good results for colored image and under intensity illumination. For such kinds of images local thresholding methods can be used like as Sauvola, Niblack etc.

\section{REFERENCES}

[1] M. Sezgin, B. Sankur, "Survey over image thresholding techniques and quantitative performance evaluation", Journal of Electronic Imaging 13 (1) (2004) 146-168.

[2] M.I. Sezan, "A peak detection algorithm and its application to histogram-based image data reduction", Computer Vision, Graphics, and Image Processing 49 (1) (1990) 36-51.

[3] Rosenfeld, P. de la Torre, "Histogram concavity analysis as an aid in threshold selection", IEEE Transactions on System, Man, and Cybernetics 13 (1983) 231-235.

[4] T. Pavlidis, "Threshold selection using second derivatives of the gray-scale image", in: Proceedings of the ICDAR, 1993, pp. 274-277.

[5]N. Otsu, "A thresholding selection method from gray-scale histogram", IEEE Transactions on System, Man, and Cybernetics 9 (1979) 62-66.

[6] J. Kittler, J. Illingworth, "Minimum error thresholding", Pattern Recognition 19 (1) (1886) 41-47.

[7] Derek Bradley, Gerhard Roth, “Adaptive Thresholding Using the Integral Image"

[8] Khurram Khurshid, Imran Siddiqi, Claudie Faure, Nicole Vincent, "Comparison of Niblack inspired Binarization methods for ancient documents", 16th International conference on Document Recognition And Retrieval, 2009.

[9] J. Sauvola, T. Seppänen, S. Haapakoski, M. Pietikänen, "Adaptive document binarization" , Fourth International Conference Document Analysis and Recognition (ICDAR), p. 147-152, Ulm, Germany, August 1997.

[10] K. Khurshid, I. Siddiqi, C. Faure, N. Vincent, "Comparison of Niblack inspired Binarization methods for ancient documents", 16th International conference on Document Recognition and Retrieval, USA, 2009.

[11] Madhuri Latha, Chakravarthy, "An Improved Bernsen Algorithm Approaches For License Plate Recognition", IOSR Journal of Electronics and Communication Engineering (IOSR-JECE) ISSN: 2278-2834, ISBN: 22788735. Volume 3, Issue 4 (Sep-Oct. 2012). 
[12] Aroop Mukherjee , Soumen Kanrar, "Enhancement of Image Resolution by Binarization" ,International Journal of Computer Applications (0975 - 8887)Volume 10- No.10, November 2010.

[13] Chien-Hsing Chou, Wen-Hsiung Lin, Fu Chang, "A binarization method with learning-built rules for document images produced by cameras",in: proceeding of ELSEVIER,2009,pp. 1518-1530

[14] Madhuri Latha.G, Chakravarthy.G, "An Improved Bernsen Algorithm Approaches For License Plate Recognition" IOSR Journal of Electronics and Communication Engineering (IOSR-JECE) ISSN: 2278-2834, ISBN: 22788735. Volume 3, Issue 4 (Sep-Oct. 2012), PP 01-05
[15] Zhang zhi-yong,song yang, "The License Plate image Binarization Based on Otsu Algorithim and Matlab Realize", IEEE 2012, International Conference on Industrial Control and Electronics Engineering

[16] Konstantinos Ntirogiannis, Basilis Gatos and and Ioannis Pratikakis, "Performance Evaluation Methodology for Historical Document Image Binarization", IEEE Transaction on Image Processing, Volume 22, No. 2, February 2013

[17]Yi wang, Bin Fang and Li-Jun Lan, " Adaptive Binarization: a New Approach to License Plate Characters Segmentation" , IEEE 2012, Proceedings of the 2012 international conference on wavelet analysis and pattern recognition, Xian, 15-17 July, 2012 